\title{
EXISTENCE AND UNIQUENESS RESULTS FOR NONLINEAR BOUNDARY VALUE PROBLEMS
}

\author{
D.D. HAI AND K. SCHMITT
}

Dedicated to Paul Waltman on the occasion of his 60th birthday

ABSTRACT. We give some elementary applications of fixed point principles to prove existence and uniqueness results for solutions of boundary value problems of ordinary and partial differential equations.

1. Introduction. In this paper we provide an elementary approach to existence and uniqueness theory for the study of nonlinear boundary value problems. The approach is based on the $L^{p}$ theory of certain linear differential operators subject to boundary constraints. In several instances we obtain, using the contraction mapping principle, improvements of, by now, classical existence and uniqueness results. Applications of variational methods and other fixed point principles to problems of this type are numerous, and we refer to [6] and [18] for results which are (in their existence part) related to ours.

The first part of the paper is devoted to nonlinear perturbations of the Laplacian, while the second part is devoted to boundary value problems for systems of ordinary differential equations.

2. Semilinear elliptic problems. Let $\Omega$ be a bounded open subset of $\mathbf{R}^{N}$ and let

$$
f: \Omega \times \mathbf{R} \times \mathbf{R}^{N} \rightarrow \mathbf{R}
$$

be a mapping satisfying Carathéodory conditions. We consider the Dirichlet problem

$$
\begin{aligned}
\Delta u & =f(x, u, \nabla u), & & x \in \Omega, \\
u & =0, & & x \in \partial \Omega .
\end{aligned}
$$

\footnotetext{
Received by the editors on March 3, 1993.

1991 Mathematics Subject Classification. 34B12.

Key words and phrases. Boundary value problems, fixed point principles, contraction mappings.

This research was supported by a research grant from NSF.

Copyright (C)1994 Rocky Mountain Mathematics Consortium
} 
In what is to follow, we shall employ the notation that $|\cdot|$ stands for absolute value in $\mathbf{R}$ and the Euclidean norm in $\mathbf{R}^{N}$ and $\|\cdot\|_{p}$ the norm in $L^{p}(\Omega)$.

We have the following results:

Theorem 1. Let $f$ satisfy

$$
\begin{gathered}
|f(x, u, v)-f(x, \tilde{u}, \tilde{v})| \leq a|u-\tilde{u}|+b|v-\tilde{v}|, \\
\forall u, \tilde{u} \in \mathbf{R}, v, \tilde{v} \in \mathbf{R}^{N},
\end{gathered}
$$

where $a$ and $b$ are nonnegative constants such that

$$
\frac{a}{\lambda_{1}}+\frac{b}{\sqrt{\lambda_{1}}}<1
$$

and $\lambda_{1}$ is the principal eigenvalue of $-\Delta$ subject to homogeneous Dirichlet boundary conditions on $\partial \Omega$. Then problem (2) has a unique solution $u \in H_{0}^{1}(\Omega) \cap H^{2}(\Omega)$.

Proof. For $v \in L^{2}(\Omega)$, let us put

$$
A v=f\left(\cdot, \Delta^{-1} v, \nabla \Delta^{-1} v\right) .
$$

By linear theory and (3), the operator $A$ is a mapping of $L^{2}(\Omega)$ to itself. We shall show that $A$ is in fact a contraction mapping. To see this, let $v_{1}, v_{2} \in L^{2}(\Omega)$. Then

$$
\begin{aligned}
\left\|A v_{1}-A v_{2}\right\|_{2} \leq & a\left\|\Delta^{-1} v_{1}-\Delta^{-1} v_{2}\right\|_{2} \\
& +b\left\|\nabla \Delta^{-1} v_{1}-\nabla \Delta^{-1} v_{2}\right\|_{2} .
\end{aligned}
$$

On the other hand, it follows from the $L^{2}$ theory of $-\Delta$ that

$$
\left\|\Delta^{-1} v_{1}-\Delta^{-1} v_{2}\right\|_{2} \leq \frac{1}{\lambda_{1}}\left\|v_{1}-v_{2}\right\|_{2},
$$

and from Green's identity that

$$
\begin{aligned}
\left\|\nabla \Delta^{-1} v_{1}-\nabla \Delta^{-1} v_{2}\right\|_{2}^{2} & \leq\left|\left(v_{1}-v_{2}, \Delta^{-1} v_{1}-\Delta^{-1} v_{2}\right)\right| \\
& \leq \frac{1}{\lambda_{1}}\left\|v_{1}-v_{2}\right\|_{2}^{2}
\end{aligned}
$$


where $(\cdot, \cdot)$ is the $L^{2}$-inner product.

Hence, combining (7) and (8) in (6) we have

$$
\left\|A v_{1}-A v_{2}\right\|_{2} \leq \frac{a}{\lambda_{1}}\left\|v_{1}-v_{2}\right\|_{2}+\frac{b}{\sqrt{\lambda_{1}}}\left\|v_{1}-v_{2}\right\|_{2} .
$$

This shows that $A$ is a contraction mapping and thus has a unique fixed point. On the other hand, if $v \in L^{2}(\Omega)$ is a fixed point of $A$, then $u=\Delta^{-1} v$ is in $H_{0}^{1}(\Omega)$ and $\Delta u \in L^{2}(\Omega)$ and $u$ solves (2).

Remark 1. It is clear from the proof that in the above the real line $\mathbf{R}$ may be replaced by $\mathbf{R}^{m}$ thus obtaining a result for systems of semilinear elliptic problems.

Remark 2. Similar results may also be derived for higher order semilinear problems of the type

$$
\begin{aligned}
\Delta^{m} u & =f\left(x, u, D^{i} u\right), \quad|i|<m, x \in \Omega, \\
u & =\frac{\partial u}{\partial n}=\cdots=\frac{\partial^{m-1} u}{\partial^{m-1} n}=0, \quad x \in \partial \Omega .
\end{aligned}
$$

In this case the contraction mapping constant is obtained by applying inequalities for higher order derivatives (see, e.g., [1] ).

Remark 3. In case $N=1$ and $\Omega=(0, T), \lambda_{1}=\pi^{2} / T^{2}$ and condition (4) becomes

$$
\frac{a T^{2}}{\pi^{2}}+\frac{b T}{\pi}<1
$$

This condition was also obtained by Mawhin [13] and earlier, using spaces of continuous functions with weighted norms by Albrecht [2].

The following result shows that linear growth conditions similar to the Lipschitz condition (3) easily yield existence results via the Schauder or similar fixed point theorems. We have:

Theorem 2. Let $f$ satisfy

$$
|f(x, u, v)| \leq a|u|+b|v|+m, \quad \forall u \in \mathbf{R}, v \in \mathbf{R}^{N},
$$


where $a$ and $b$ are nonnegative constants such that (4) holds and, again, $\lambda_{1}$ is the principal eigenvalue of $-\Delta$ subject to homogeneous Dirichlet boundary conditions on $\partial \Omega$, and $m$ is a nonnegative $L^{2}$ function. Then problem (2) has a solution $u \in H_{0}^{1}(\Omega) \cap H^{2}(\Omega)$.

Proof. Define the nonlinear operator $A$ as in the proof of Theorem 1. Then $A$ is completely continuous, as follows from linear theory, and calculations similar to those used in the proof of that theorem show that

$$
\|A v\|_{2} \leq a\left\|\Delta^{-1} v\right\|_{2}+b\left\|\nabla \Delta^{-1} v\right\|_{2}+\|m\|_{2} .
$$

Using (7) and (8), we then obtain

$$
\|A v\|_{2} \leq \frac{a}{\lambda_{1}}\|v\|_{2}+\frac{b}{\sqrt{\lambda_{1}}}\|v\|_{2}+\|m\|_{2} .
$$

It follows from (4) and (6) that one can find a closed ball in $L^{2}(\Omega)$ which is mapped into itself by $A$, proving the existence of at least one fixed point of $A$ by the Schauder fixed point theorem.

3. Systems of ordinary differential equations. In this section we shall consider the nonlinear boundary value problem

$$
\begin{aligned}
u^{\prime \prime}+K u^{\prime}+f\left(x, u, u^{\prime}\right) & =0, & & 0<x<T, \\
u & =0, & & x \in\{0, T\},
\end{aligned}
$$

where

$$
f:[0, T] \times H \times H \rightarrow H
$$

and $H$ is a Banach space with norm $|\cdot|$.

Theorem 3. Assume that the mapping $f$ is continuous and satisfies the Lipschitz condition

$$
\begin{gathered}
|f(x, u, v)-f(x, \tilde{u}, \tilde{v})| \leq a|u-\tilde{u}|+b|v-\tilde{v}| \\
\forall u, \tilde{u} \in H, v, \tilde{v} \in H, x \in(0, T)
\end{gathered}
$$


where

$$
a \phi(K)+b \psi(K)<1
$$

and $\phi, \psi$ are given by

$$
\phi(K)=\frac{1}{|K|\left(1-e^{-|K| T}\right)} \int_{0}^{T}\left(1-e^{-|K| s}\right)\left(1-e^{-|K|(T-s)}\right) d s
$$

and

$$
\psi(K)=\frac{2}{|K|} \frac{\left(1-e^{-|K| T / 2)^{2}}\right.}{1-e^{-|K| T}} .
$$

Then problem (14) has a unique solution.

Proof. We observe that $u$ is a solution of (14) if and only if $v=u^{\prime}$ solves

$$
\begin{aligned}
v^{\prime}+K v+f\left(x, \int_{0}^{x} v, v\right) & =0, \quad 0<x<T, \\
\int_{0}^{T} v & =0 .
\end{aligned}
$$

We proceed thus to establish the existence of a unique solution of (20).

We first assume that $K>0$. Let $M$ be the subspace of $L^{1}((0, T) ; H)$ consisting of those $v$ with $\int_{0}^{T} v=0$. Define $A: M \rightarrow M$ by

$$
\begin{aligned}
A v(x)= & \frac{K e^{-K x}}{1-e^{-K T}} \int_{0}^{T} e^{-K x}\left(\int_{0}^{x} e^{K s} N v(s)\right) \\
& -e^{-K x} \int_{0}^{x} e^{K s} N v(s),
\end{aligned}
$$

where $N$ is the Nemitskii operator

$$
N v(x)=f\left(x, \int_{0}^{x} v, v\right)
$$


Then $v$ is a solution of (20) if and only if $v$ is a fixed point of $A$.

For $u, v \in M$, let $w=u-v$ and $p(x)=|N u(x)-N v(x)|$. Then

$$
p(x) \leq a\left|\int_{0}^{x} w\right|+b|w(x)|, \quad x \in[0, T]
$$

We next rewrite (21) as

$$
\begin{aligned}
A v(x)=\frac{e^{-K x}}{1-e^{-K T}}\left\{\int_{0}^{x}\left(1-e^{K s}\right)\right. & N v(s) \\
& \left.+\int_{x}^{T}\left(1-e^{-K(T-s)}\right) N v(s)\right\},
\end{aligned}
$$

from which follows

$$
\int_{0}^{\pi}|A u-A v| \leq 2 \int_{0}^{T} \frac{\left(1-e^{-K s}\right)\left(1-e^{-K(T-s)}\right)}{K\left(1-e^{-K T}\right)} p(s) .
$$

On the other hand, since $\int_{0}^{T} w=0$, it follows that $\left|\int_{0}^{x} w\right| \leq$ $(1 / 2) \int_{0}^{T}|w|, x \in[0, T]$, further the function $\left(1-e^{-K s}\right)\left(1-e^{-K(T-s)}\right)$ assumes its maximum at $T / 2$. Using these facts in (23), we obtain that

$$
\|A u-A v\|_{1} \leq(a \phi(K)+b \psi(K))\|u-v\|_{1},
$$

and hence that $A$ is a contraction mapping on $M$ and therefore has a unique fixed point there.

If $K<0$, we apply what has just been proved with $f(\pi-x, u,-v)=$ $g(x, u, v)$ in place of $f(x, u, v)$.

Remark 4. Letting $K \rightarrow 0$ in (18) and (19), we see that this inequality requirement becomes

$$
\frac{a T^{2}}{6}+\frac{b T}{2}<1
$$

which is not as good as the requirements stated earlier in Remark 3. The next result, however, shows that, with the aid of Opial's inequality [14] and [15], we may obtain an improvement containing known inequality requirements. 
We shall now consider problem (14) with $K=0$, i.e.,

$$
\begin{aligned}
u^{\prime \prime}+f\left(x, u, u^{\prime}\right) & =0, & & 0<x<T, \\
u & =0, & & x \in\{0, T\} .
\end{aligned}
$$

Theorem 4. Let $H$ be a Hilbert space and assume that the mapping $f$ is continuous and satisfies the Lipschitz condition

$$
\begin{gathered}
|f(x, u, v)-f(x, \tilde{u}, \tilde{v})| \leq a|u-\tilde{u}|+b|v-\tilde{v}|, \\
\forall u, \tilde{u} \in H, v, \tilde{v} \in H, x \in(0, T),
\end{gathered}
$$

where

$$
\frac{a^{2} T^{4}}{\pi^{4}}+\frac{b^{2} T^{2}}{\pi^{2}}+\frac{a b T^{3}}{2 \pi^{2}}<1 .
$$

Then problem (24) has a unique solution.

Proof. In what follows we shall need the following result due to Opial [15]. If $u \in W_{0}^{1,2}((0, T) ; H)$, then

$$
\int_{0}^{T}|u(x)|\left|u^{\prime}(x)\right| \leq \frac{T}{4} \int_{0}^{T}\left|u^{\prime}(x)\right|^{2} .
$$

We let $V$ be the subspace of $L^{2}((0, T) ; H)$ consisting of functions of zero mean. Then $V$ is a Banach space. We define $A: V \rightarrow V$ by

$$
A v(x)=\frac{1}{T} \int_{0}^{T} \int_{0}^{s} N v(t)-\int_{0}^{x} N v(t),
$$

where $N$ again is the Nemitskii operator

$$
N v(x)=f\left(x, \int_{0}^{x} v, v\right) .
$$

We show that $A$ is a contraction mapping on $V$. To see this, let $u, v \in V$, then by Wirtinger's inequality

$$
\|A u-A v\|_{2} \leq \frac{T}{\pi}\|N u-N v\|_{2} .
$$


Since $f$ satisfies the Lipschitz condition (25) we get that

$$
|N u(s)-N v(s)| \leq a\left|\int_{0}^{s}(u-v) d \tau\right|+b|u(s)-v(s)|,
$$

for every $s \in[0, T]$, which implies by Wirtinger's and Opial's inequalities that

$$
\begin{aligned}
\|A u-A v\|_{2}^{2} \leq & \frac{a^{2} T^{4}}{\pi^{4}}\|u-v\|_{2}^{2}+\frac{b^{2} T^{2}}{\pi^{2}}\|u-v\|_{2}^{2} \\
& +\frac{a b T^{3}}{2 \pi^{2}}\|u-v\|_{2}^{2} .
\end{aligned}
$$

That $A$ is a contraction mapping now follows from (26).

Remark 5. Let us compare what has been obtained with classical results. Picard obtained the inequality requirement

$$
\frac{a T^{2}}{8}+\frac{b T}{2}<1
$$

(see $[\mathbf{1 1}]$ and $[\mathbf{1 6}]$ ), which was generalized by Lettenmeyer $[\mathbf{1 2}]$

$$
\frac{a T^{2}}{\pi^{2}}+4 \frac{b T}{\pi^{2}}<1
$$

This result was further extended by Coles and Sherman $[\mathbf{7}]$

$$
\begin{gathered}
a T^{2}+4 b T<12, \quad \text { for } a \leq b^{2} \\
(\sqrt{3}-1) a T^{2}+2 b T<4 \sqrt{3}, \quad \text { for } a>b^{2} .
\end{gathered}
$$

All of these inequality requirements are not as good as (10), except (33) which in a small region of the $a T^{2}-b T$ plane is better than (10). A quick plot will convince the reader. The inequality requirement given by (26), however, includes all of the above.

Much larger existence and uniqueness regions for problem (24), however, have been known for some time. We refer the interested reader to the conditions given in $[\mathbf{4}, \mathbf{8}, \mathbf{9}, \mathbf{1 7}]$. As remarked in $[\mathbf{3}]$ one cannot expect that successive approximations will converge in these larger regions. 
4. Further existence results. In the following we shall fix the $x-\operatorname{interval} I=[0, \pi]$.

Theorem 5. Suppose that

(i) $f: I \times H \times H \rightarrow H$ is completely continuous.

(ii) There exist real numbers $a, b \geq 0, R>0$ and nondecreasing functions $F, G$ and $J: \mathbf{R}^{+} \rightarrow \mathbf{R}^{+}$with

$$
\left[a F(\sqrt{\pi} R)+\frac{b \pi}{4} G(\sqrt{\pi} R)\right] R^{2}+\sqrt{\pi} R J(\sqrt{\pi} R) \leq R^{2}
$$

such that

$$
(u, f(x, u, v)) \leq a|u|^{2} F(|u|)+b|u||v| G(|u|)+|u| J(|u|),
$$

for all $x \in I$ and $u, v \in H$ with $|u| \leq \sqrt{\pi} R$.

(iii) There exists a continuous function $h: \mathbf{R}^{+} \rightarrow \mathbf{R}^{+} \backslash\{0\}$ and constants $C$ and $K$ such that

$$
\mid\left(v, f(x, u, v) \mid \leq h\left(|v|^{2}\right)\left(|v|^{2}+C\right),\right.
$$

for all $x \in I$ and $u, v \in H$ with $|u| \leq \sqrt{\pi} R$, and

$$
\int_{M}^{K} \frac{d s}{h(s)+|K|}>2\left(R^{2}+\pi \tilde{C}\right)
$$

where

$$
\tilde{C}=\frac{1}{4}\left(\frac{K+C}{\sqrt{K}}\right)^{2}, \quad M=\frac{R^{2}}{\pi} .
$$

Then the problem (14) has at least one solution.

Proof. Define $g: I \times H \times H \rightarrow H$ by

$$
g(x, u, v)=f(x, u, P v)
$$

where

$$
P v= \begin{cases}v & \text { if }|v| \leq \sqrt{K} \\ \frac{\sqrt{K} v}{|v|} & \text { if }|v|>\sqrt{K}\end{cases}
$$


Then $g$ is completely continuous and

$$
\begin{gathered}
(u, g(x, u, v)) \leq a|u|^{2} F(|u|+b|u||v| G(|u|)+|u| J(|u|) \\
\mid\left(v, g(x, u, v) \mid \leq \tilde{h}\left(|v|^{2}\right)\left(|v|^{2}+\tilde{C}\right)\right.
\end{gathered}
$$

for all $x \in I$ and $u, v \in H$ with $|u| \leq \sqrt{\pi} R$, where $\tilde{h}(x)=h(\min (x, K))$. Let $E=\left\{u \in C(I ; H): \int_{0}^{\pi} u=0\right\}$ with $L^{2}$-inner product. Define $A: E \rightarrow E$ by (21) with $N u(s)=g\left(s, \int_{0}^{s} u, u(s)\right)$. Then $A$ is completely continuous and $A v=u$ if and only if

$$
u^{\prime}+K u+g\left(x, \int_{0}^{x} v, v\right)=0, \quad \int_{0}^{\pi} u=0 .
$$

We claim that $u \neq \lambda A u$ for $u \in E$ with $\|u\|_{2}=R$ and $\lambda \in(0,1)$. Suppose the contrary, and let $u \in E,\|u\|_{2}=R$ and $\lambda \in(0,1)$ such that

$$
u=\lambda A u
$$

or

$$
\begin{aligned}
u^{\prime}+K u+\lambda g\left(x, \int_{0}^{x} u, u\right) & =0 \\
\int_{0}^{\pi} u & =0 .
\end{aligned}
$$

Taking the inner product of (40) with $\int_{0}^{x} u$ and integrating gives

$$
\|u\|_{2}^{2}=\lambda\left(\int_{0}^{x} u, g\left(x, \int_{0}^{x} u, u\right)\right) .
$$

By (34),

$$
\begin{aligned}
\left(\int_{0}^{x} u, g\left(x, \int_{0}^{x} u, u\right)\right) \leq & a\left|\int_{0}^{x} u\right|^{2} F\left(\left|\int_{0}^{x} u\right|\right) \\
& +b\left|\int_{0}^{x} u\right| G\left(\left|\int_{0}^{x} u\right|\right)|u(x)| \\
& +\left|\int_{0}^{x} u\right| J\left(\left|\int_{0}^{x} u\right|\right)
\end{aligned}
$$


for every $x \in I$, since $\left|\int_{0}^{x} u\right| \leq \sqrt{\pi}\|u\|_{2}=\sqrt{\pi} R$. Combining (41), (42) gives, by Poincaré's and Opial's inequalities

$$
R^{2}=\|u\|_{2}^{2} \leq \lambda\left[a F(\sqrt{\pi} R)+\frac{b \pi}{4} G(\sqrt{\pi} R)\right] R^{2}+\sqrt{\pi} R J(\sqrt{\pi} R)<R^{2}
$$

a contradiction, proving the claim. It then follows from the LeraySchauder continuation principle that $A$ has a fixed point $v$ with $\|v\|_{2} \leq$ $R$. Hence, $u(x)=\int_{0}^{x} v$ is a solution of

$$
u^{\prime \prime}+K u^{\prime}+g\left(x, u, u^{\prime}\right)=0, \quad u(0)=u(\pi)=0 .
$$

We verify now that $\left\|u^{\prime}\right\|_{0} \leq \sqrt{K}$. Taking the inner product of (43) with $u^{\prime}$ gives

$$
\frac{1}{2} \frac{d}{d x}\left|u^{\prime}(x)\right|^{2}+K\left|u^{\prime}(x)\right|^{2}+\left(u^{\prime}, g\left(x, u, u^{\prime}\right)\right)=0 .
$$

which implies

$$
\begin{aligned}
&\left.\left|\frac{d}{d x}\right| u^{\prime}(x)\right|^{2} \mid \leq 2|K|\left|u^{\prime}(x)\right|^{2}+2\left|\left(u^{\prime}(x), g\left(x, u, u^{\prime}\right)\right)\right| \\
& \leq 2\left(P\left(\left|u^{\prime}(x)\right|^{2}\right)+|K|\right)\left(\left|u^{\prime}(x)\right|^{2}+\tilde{C}\right) \\
&\left|\frac{d}{d x} \int^{\left|u^{\prime}(x)\right|^{2}} \frac{d s}{\tilde{h}(s)+|K|}\right| \leq 2\left(\left|u^{\prime}(x)\right|^{2}+\tilde{C}\right), \quad x \in I .
\end{aligned}
$$

Since $\left\|u^{\prime}\right\|_{2} \leq R$, there exists $x_{0} \in I$ such that

$$
\left|u^{\prime}\left(x_{0}\right)\right|^{2} \leq R^{2} / \pi .
$$

From (46) and (47), we deduce

$$
\begin{aligned}
\int_{0}^{\left|u^{\prime}(x)\right|^{2}} \frac{d s}{\tilde{h}(s)+|K|} & \leq \int_{0}^{R^{2} / \pi} \frac{d s}{\tilde{h}(s)+|K|}+2\left(\|\left. u^{\prime}\right|_{2} ^{2}+\pi \tilde{C}\right) \\
& \leq \int_{0}^{K} \frac{d s}{\tilde{h}(s)+|K|}
\end{aligned}
$$


since

$$
\int_{R^{2} / \pi}^{K} \frac{d s}{\tilde{h}(s)+|K|}=\int_{R^{2} / \pi}^{K} \frac{d s}{h(s)+|K|} \geq 2\left(R^{2}+\pi \tilde{C}\right),
$$

by (35). Hence $\left|u^{\prime}(x)\right|^{2} \leq K$ for every $x \in I$ and so $u$ is a solution of (14) by definition of $g$.

This completes the proof of Theorem 5 .

Remark 6. (i) The conclusion of Theorem 5 holds if $H=\mathbf{R}^{N}$ and (i) is replaced by the Carathéodory condition, i.e., for each $(u, v) \in H \times H$, the mapping $x \mapsto f(x, u, v)$ is measurable, for a.e. $x \in I$, the mapping $(u, v) \mapsto f(x, u, v)$ is continuous, and for each $r>0$, there exists $g_{r} \in L^{1}(I ; \mathbf{R})$ such that

$$
|f(x, u, v)| \leq g_{r}(x)
$$

for a.e. $x \in I$ and $u, v \in H$ with $|u| \leq r,|v| \leq r$.

(ii) Let $F(x)=G(x)=1$ and $J(x)=1$ for every $x \in I$. Then condition (34) becomes

$$
a+\frac{b \pi}{4}<1 .
$$

The above result is an extension of a result of Mawhin [13] who proved an existence result for (14) for completely continuous $f$ satisfying the following conditions.

a) There exist nonnegative numbers $a, b$ and $c$ with $a+b<1$, such that

$$
(u, f(x, u, v)) \leq a|u|^{2}+b|u||v|+c|u|
$$

for all $x \in I$ and $u, v \in H$.

b) There exists a continuous function $h: \mathbf{R}^{+} \rightarrow \mathbf{R}^{+} \backslash\{0\}$ such that $h+2|K|$ is a two-Nagumo function, i.e.,

$$
\int_{0}^{\infty} \frac{d s}{h(s)+2|K|}=\infty
$$

and such that

$$
2 \mid\left(x,\left.f(x, u, v)\left|\leq h\left(|v|^{2}\right)\right| v\right|^{2}\right.
$$


for all $x \in I$ and $u, v \in H$ with $|u| \leq \pi(1-a-b)^{-1} c$.

Next we shall establish an existence and uniqueness result for (14) under a mere continuity assumption of $f$.

\section{Theorem 6. Suppose that}

(i) $f: I \times H \times H \rightarrow H$ is continuous and for each $r>0$, there exists $g_{r} \in L^{1}$ such that

$$
|f(x, u, v)| \leq g_{r}(x)
$$

for all $x \in I$ and $u, v \in H$ with $|u| \leq r,|v| \leq r$.

(ii) There exist nonnegative numbers $a$ and $b$ with $a+b \pi / 4<1$, such that

$$
(u-\tilde{u}, f(x, u, v)-f(x, \tilde{u}, \tilde{v})) \leq a|u-\tilde{u}|^{2}+b|u-\tilde{u}||v-\tilde{v}|
$$

for all $x \in I$ and $u, v, \tilde{u}, \tilde{v} \in H$.

(iii) $f$ satisfies condition (iii) of Theorem 5 with $R=(1-a-$ $b \pi / 4)^{-1} c$, where $c=\|f(x, 0,0)\|_{2}$.

Then the problem (14) has exactly one solution.

Proof. Define $g: I \times H \times H$ by (36). Then

$$
(u-\tilde{u}, g(x, u, v)-g(x, \tilde{u}, \tilde{v})) \leq a|u-\tilde{u}|^{2}+b|u-\tilde{u}||v-\tilde{v}|
$$

for all $x \in I$ and $u, v, \tilde{u}, \tilde{v} \in H$.

Let $E$ be the subspace of $L^{2}(I ; H)$ consisting of $u$ with $\int_{0}^{\pi} u=0$. Then $E$ is a Hilbert space.

Define $A: E \rightarrow E$ by

$$
A u(x)=\frac{1}{\pi} \int_{0}^{\pi} \int_{0}^{t} N u(s) d s d t-\int_{0}^{x} N u(s) d s
$$

where $N u(x)=K u(x)+g\left(x, \int_{0}^{x}, u, u(x)\right)$. Then, using assumption (i), we see that $A$ is continuous. Let $u, v \in E$; then we have, by inverting 
the order of integration,

$$
\begin{aligned}
(A u-A v, u-v) & =-\int_{0}^{\pi}\left(\int_{0}^{t}(N u-N v) d s, u(t)-v(t)\right) d t \\
& =\int_{0}^{\pi}\left(\int_{0}^{s}(u-v) d t, N u(s)-N v(s)\right) d s
\end{aligned}
$$

Since $\int_{0}^{\pi}(u-v)=0$, which implies by (48) and Poincaré's inequality that

$$
(A u-A v, u-v) \leq\left(a+b \frac{\pi}{4}\right)\|u-v\|_{2}^{2} .
$$

Define $B: E \rightarrow E$ by $B v=v-A v$. Then

$$
(B u-B v, u-v) \geq\left(1-\left(a+\frac{b \pi}{4}\right)\right)\|u-v\|_{2}^{2} \quad \text { for } u, v \in E .
$$

Hence, it follows from the Minty-Browder theorem (see, e.g., [5]) that there exists a unique $u \in E$ such that $B u=0$, i.e., $A$ has a unique fixed point on $E$. We claim that $\|u\|_{2} \leq R$. Indeed, letting $v=0$ in (49) gives

$$
(B u, u) \geq\left(1-\left(a+\frac{b \pi}{4}\right)\right)\|u\|_{2}^{2}-\|f(x, 0,0)\|_{2}\|u\|_{2}>0
$$

for $\|u\|_{2}>R$.

Hence $\|u\|_{2} \leq R$, as claimed.

Proceeding as in the proof of Theorem 5, it follows that $u(x)=\int_{0}^{x} v$ is the unique solution of (17).

Remark 7. The virtue of Theorem 6 is that $f$ may take a bounded subset in $H$ into an unbounded subset.

\section{REFERENCES}

1. S. Agmon, Lectures on elliptic boundary value problems, Van Nostrand, Princeton, 1965.

2. J. Albrecht, Zur Wahl der Norm beim Iterationsverfahren für Randwertaufgaben, ZAMM 52 (1972), 626-628. 
3. P. Bailey, On the interval of convergence of Picard's iteration, ZAMM 48 (1968), 127-128.

4. P. Bailey, L. Shampine and P. Waltman, Nonlinear two point boundary value problems, Academic Press, New York, 1968.

5. H. Brezis, Analyse fonctionelle, théorie et applications, Masson, Paris, 1983.

6. A. Castro, A semilinear Dirichlet problem, Canad. J. Math. 31 (1979), $337-340$.

7. W. Coles and T. Sherman, Convergence of successive approximations for nonlinear two point boundary value problems, SIAM J. Appl. Math. 15 (1967), 426-433.

8. H. Epheser, Über die Existenz der Lösungen von Randwertaufgaben mit gewöhnlichen nichtlinearen Differentialgleichungen zweiter Ordnung, Math. Z. 61 (1955), 435-454.

9. C. Fabry and P. Habets, The Picard boundary value problem for nonlinear second orer vector differential equations, J. Differential Equations 42 (1981), 186-198.

10. D.D. Hai, Existence and uniqueness of solutions for a nonlinear second order differential equation in Hilbert space, Proc. Edinburgh Math. Soc. 33 (1990), 89-95.

11. P. Hartman, Ordinary differential equations, J. Wiley and Sons, New York, 1964.

12. F. Lettenmeyer, Über die von einem Punkt ausgehenden Integralkurven einer Differentialgleichung zweiter Ordnung, Deutsche Math. 7 (1944), 56-74.

13. J. Mawhin, Two point boundary value problems for nonlinear second order differential equations in Hilbert space, Tôhoku Math. J. 32 (1980), 225-233.

14. C. Olech, A simple proof of a certain result of Z. Opial, Ann. Polon. Math. 8 (1960), 61-63.

15. Z. Opial, Sur un inegalité, Ann. Polon. Math. 8 (1960), 29-32.

16. É. Picard, Leçons sur quelques problèmes aux limites de la théorie des équations différentielles, Gauthier-Villars, Paris, 1930.

17. C. de la Vallée Poussin, Sur l'équation différentielle linéaire du second ordre, J. Math. Pures Appl. 94 (1929), 125-144.

18. S.A. Williams, A nonlinear elliptic boundary value problem, Pacific J. Math. 44 (1973), 767-774.

Department of Mathematics, University of Utah, Salt lake City, UT 84112 